\title{
Noninvasive Ventilation in Mustard Airway Diseases
}

\author{
Rasoul Aliannejad MD, Mohammadreza Peyman MD, and Mostafa Ghanei MD
}

\begin{abstract}
BACKGROUND: Noninvasive ventilation (NIV) has been shown to improve numerous clinical measures in subjects with COPD, including gas exchange, exercise tolerance, dyspnea, hospitalizations, health-related quality of life (HRQOL), and functional status. Although useful for classical COPD, NIV has not been validated specifically as a treatment option for mustard airway disease. Our aim was to assess the efficacy of NIV in subjects with severe mustard airway disease. METHODS: This study was performed over the course of 12 months on 20 subjects with severe mustard airway disease (Global Initiative for Chronic Obstructive Lung Disease criteria). Subjects were selected from the pulmonary clinic of Baqiyatallah Hospital in Tehran, Iran. Body mass index, lung function, dyspnea, exercise tolerance, HRQOL, sleep quality, and hospitalization measures were assessed before and after NIV treatment. RESULTS: NIV improved HRQOL $(P=.03)$ and sleep quality $(P=.06)$ in very severe subjects and reduced hospitalizations $(P=.003)$ and exacerbations $(P=.01)$ in severe subjects. Chest imaging studies showed that these subjects had heterogeneous airway disease, the main finding of which was bronchiolitis obliterans. CONCLUSIONS: NIV can be used as an effective new palliative method in the treatment of mustard airway disorders. Subjects with very severe and severe disease benefitted differently from NIV. Key words: noninvasive ventilation (NIV); sulfur mustard; chronic obstructive respiratory disease (COPD); bronchiolitis obliterans. [Respir Care 2015;60(9):1324-1329. (C) 2015 Daedalus Enterprises]
\end{abstract}

\section{Introduction}

Noninvasive ventilation (NIV) is a well-established treatment for chronic hypercapnic respiratory failure in subjects with neuromuscular disease or skeletal deformity. There is increasing interest in its use for subjects with chronic respiratory failure due to COPD. ${ }^{1}$

The authors are affiliated with the Respiratory Diseases Research Center, Janbazan Medical and Engineering Research Center, Tehran, Iran. Dr Aliannejad is also affiliated with the Pulmonary Department, Shariati Hospital, Tehran University of Medical Sciences, Tehran, Iran. Drs Peyman and Ghanei are also affiliated with the Chemical Injuries Research Center, Baqiyatallah University of Medical Sciences, Tehran, Iran.

Dr Ghanei presented a version of this paper at the 20th Annual Congress of the European Respiratory Society, held September 18-22, 2010, in Barcelona, Spain.

The authors have disclosed no conflicts of interest.

Correspondence: Mostafa Ghanei MD, Baqiyatallah University of Medical Sciences, Mollasadra Avenue, Tehran 1411713135, Iran. E-mail: mghaneister@gmail.com,m.ghanei@bmsu.ac.ir.

DOI: $10.4187 /$ respcare. 02900
A systematic review affirmed that bi-level NIV use in a selected population of subjects with severe stable COPD can improve gas exchange, exercise tolerance, dyspnea, health-related quality of life (HRQOL), and functional status while also reducing work of breathing (due to lung hyperinflation) and hospitalizations. ${ }^{2}$

Approximately 34,000 presently living Iranians were exposed to mustard agent during the Iran-Iraq war (1980 1988), $42.5 \%$ of which have respiratory problems. Of those individuals with respiratory problems, $1 \%$ are classified as severe. Approximately $95 \%$ are males who were between 17 and $30 \mathrm{y}$ of age at the time of exposure. ${ }^{3}$ The subjects enrolled in this study had severe obstructive pulmonary disease referred to as mustard airway disease, a late complication of mustard gas exposure. Previous pathological and radiological studies have shown bronchiolitis obliterans $^{4,5}$ to be the main airway disease in mustard gas-exposed cases, and imaging studies also indicated other airway diseases such as bronchiectasis, tracheobronchomalacia, and tracheobronchial stenosis. ${ }^{6-8}$ Not surprisingly, sulfur mustard can affect the entire set of conducting airways and the small airways in particular. Because our pathological and radiological findings demonstrated that 
mustard airway disease differs significantly from classical COPD, this study was designed to assess the efficiency of NIV in this new untested group of subjects with obstructive pulmonary disease.

\section{Methods}

\section{Subject Selection}

Twenty subjects with severe mustard airway disease at the pulmonary clinic of Baqiyatallah Hospital were enrolled between 2005 and 2009. This study was approved by the human research committee at the Janbazan Medical and Engineering Research Center. All subjects gave written informed consent. Inclusion criteria were: $<75 \mathrm{y}$ of age, $\mathrm{FEV}_{1}<50 \%$ of predicted, $\mathrm{FEV}_{1} / \mathrm{FVC}<0.70$, and a stable clinical state (no exacerbation for 3 months before initiation of the study). Exclusion criteria were: $15 \%$ increase in $\mathrm{FEV}_{1}$ after administration (2 puffs) of an inhaled bronchodilator, any contraindication to NIV such as tracheobronchial stenosis, important comorbidities, or a current smoking history.

In addition, chest high-resolution computed tomography and fiberoptic bronchoscopy of our subjects (from the hospital data set) were evaluated for case selection and to determine the type of airway disease. The results of arterial blood gas tests were not used as criteria for enrollment. All subjects received the standard level of pulmonological care, including inhaled medications, oral $\mathrm{N}$-acetylcysteine (600 mg every $12 \mathrm{~h}$ ), and strong encouragement to remain physically active in addition to antibiotic treatment, systemic steroid treatment, and hospitalization as indicated by an internist. Prescribed inhalers included fluticasone propionate/salmeterol (250 $\mu \mathrm{g}, 2$ puffs every $12 \mathrm{~h}$ ), ipratropium bromide ( 4 puffs 4 times/d), and salbutamol (2-4 puffs as needed).

\section{Study Design}

This study spanned 12 months. After inclusion, subjects were admitted to the hospital and instructed to use a ventilator overnight and for at least $4 \mathrm{~h}$ daily throughout the entire study period. All subjects were acclimatized to NIV in the hospital for a period of $7 \mathrm{~d}$. During hospitalization, subjects were instructed on how to use the NIV equipment properly, and the ventilator settings were adjusted for comfort and maximal gas exchange. After discharge, a general physician visited all subjects at home, and ICU nurses visited subjects every week for the first 2 months, every 2 weeks for the second 2 months, and every month for the remainder of the study to encourage compliance. A technical assistance telephone number was also provided to all subjects as a precaution.

\section{QUICK LOOK}

\section{Current knowledge}

Noninvasive ventilation (NIV) is first-line treatment for COPD exacerbations, improving outcomes and reducing costs. The use of NIV in other disease states has met with varying degrees of success.

\section{What this paper contributes to our knowledge}

In a small group of subjects with mustard agent exposure and obstructive disease, the use of NIV for $4 \mathrm{~h}$ daily and overnight improved health-related quality of life and sleep quality in those subjects with very severe mustard airway disease. Subjects with severe mustard airway disease had reduced hospitalizations and respiratory exacerbations. NIV did not significantly improve exercise tolerance, pulmonary function, or dyspnea.

\section{Assessments}

Personnel blinded to treatment and not involved in the study analysis performed all physiological measurements. The following assessments were made at the onset and conclusion of the study.

Lung and Respiratory Muscle Function. Spirometry was performed using a constant-volume body plethysmograph (Erich Jaeger, Friedberg, Germany) following the guidelines of the American Thoracic Society. ${ }^{9}$ Static pulmonary volumes (residual volume, functional residual capacity, and total lung capacity) were measured using standard methods. ${ }^{10}$ Diffusion capacity was measured by pulmonary transfer of carbon monoxide. ${ }^{11}$ Arterial blood was sampled at the radial artery while subjects were in a semirecumbent position while they breathed both ambient air and their usual oxygen flow. $\mathrm{P}_{\mathrm{aO}_{2}}, \mathrm{P}_{\mathrm{aCO}_{2}}$, and $\mathrm{pH}$ were measured via an automated analyzer.

Dyspnea and Exercise Tolerance. Resting dyspnea was assessed using the visual analog and Borg dyspnea scales. ${ }^{12}$ Exercise capacity was assessed by the 6-min walk test while subjects breathed ambient air. ${ }^{13}$

Verbal encouragement was given at definite intervals during the test. Three practice tests were performed on each of 2 consecutive d, and the highest value was recorded. Arterial oxygen saturation was monitored throughout the 6-min walk test using a pulse oximeter, and the lowest value was recorded.

HRQOL. HRQOL was assessed using the Persian translation of the St George Respiratory Questionnaire. ${ }^{14}$ 


\section{Noninvasive Ventilation in Mustard Airway Diseases}

Sleep Quality. Sleep quality was assessed with the Persian translation of the Pittsburgh Sleep Quality Questionnaire. ${ }^{15}$

Hospitalizations. The number and locations of hospital and ICU admissions and episodes of endotracheal intubation in the previous $3 \mathrm{y}$ were recorded. These data were obtained by interviews with the subjects on admission, from the hospital registry, and by interviews with the subjects' relatives or general practitioners.

The number of respiratory exacerbations in the previous 3 y was recorded. Exacerbation was defined as a change in the rate, character, or color of cough and sputum production or increased dyspnea requiring medical evaluation. All hospital admissions, endotracheal intubations, and pulmonary or extrapulmonary causes of death at 4,8 , and 12 months were recorded.

Treatment Compliance. Time spent on NIV was recorded at each visit during the follow-up period. Total time of ventilator use was obtained from ventilator memory cards.

Ventilatory Support. The modality of NIV was pressure support ventilation delivered by a BiPAP Pro 2 system (Philips Respironics, Murrysville, Pennsylvania) via a nasal mask (Philips Respironics). The mask was of adequate size to fit each patient's nose. A one-way circuit was provided with a plateau exhalation valve to avoid rebreathing. ${ }^{16}$ The ventilator was set in spontaneous mode at the maximal tolerated inspiratory positive airway pressure (IPAP) and at an expiratory positive airway pressure as tolerated at $4 \mathrm{~cm} \mathrm{H}_{2} \mathrm{O}$ with Bi-Flex modality. Some precautions for the use of high IPAP were taken that are specific to our subjects. Many subjects had skin and corneal scarring due to sulfur mustard exposure, nasal mucosa dryness due to lachrymal canal ligation surgery (to prevent conjunctiva dryness), or a history of corneal graft surgery. Furthermore, subjects worried about air leakage through masks and the potential for corneal injury. As a result, IPAP was set mainly according to subject comfort. Low IPAP was used with prudence first because of our experience and because of the novelty of our application of NIV. Oxygen was also added to the mask side port at a flow necessary to achieve a target oxygen saturation of $>90 \%$.

Body Mass. Body mass index was calculated before and 12 months after starting NIV treatment.

\section{Statistical Analysis}

Statistical analysis of all data was performed with the Wilcoxon signed-rank test, with the level of significance set at $P<.05$.
Table 1. Baseline Clinical and Physiological Characteristic of the 20 Subjects

\begin{tabular}{|c|c|}
\hline Characteristic & Values \\
\hline Age, y & $43 \pm 5$ \\
\hline BMI, $\mathrm{kg} / \mathrm{m}^{2}$ & $24.5 \pm 4.3$ \\
\hline Borg dyspnea scale score & $6.1 \pm 1.1$ \\
\hline Visual analog dyspnea scale score & $7.0 \pm 1.5$ \\
\hline $\mathrm{FEV}_{1}, \%$ predicted & $25 \pm 9$ \\
\hline Vital capacity, $\%$ predicted & $51 \pm 1$ \\
\hline TLC, $\%$ predicted & $108 \pm 39$ \\
\hline $\mathrm{RV}, \%$ predicted & $250 \pm 120$ \\
\hline RV/TLC, \% predicted & $213 \pm 45$ \\
\hline FRC, $\%$ predicted & $150 \pm 48$ \\
\hline $\mathrm{R}_{\mathrm{aw}}, \%$ predicted & 846.76 \\
\hline $\mathrm{T}_{\mathrm{LCO}}, \%$ predicted & $107 \pm 23$ \\
\hline $\mathrm{P}_{\mathrm{O}_{2}}, \mathrm{~mm} \mathrm{Hg}$ & $60 \pm 2$ \\
\hline $\mathrm{P}_{\mathrm{CO}_{2}}, \mathrm{~mm} \mathrm{Hg}$ & $43 \pm 9$ \\
\hline $\mathrm{pH}$ & $7.36 \pm 0.03$ \\
\hline 6MWD, m & $177.4 \pm 1.4$ \\
\hline PSQI score & $13 \pm 4$ \\
\hline No. of hospitalizations/y & $2.1 \pm 1.5$ \\
\hline No. of ICU admissions/y & $0.07 \pm 0.27$ \\
\hline No. of intubations/y & 0 \\
\hline No. of exacerbations/y & $3.1 \pm 4.0$ \\
\hline 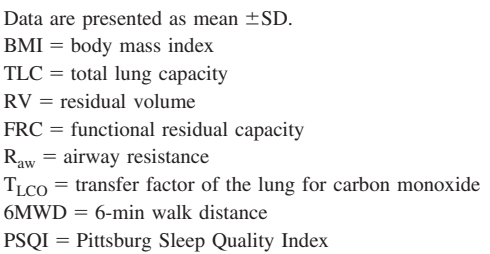 & \\
\hline
\end{tabular}

\section{Results}

All subjects were male, with an average age of $43 \pm 5 y$ (mean $\pm \mathrm{SD}$ ). All 20 subjects completed the 12-month course of domiciliary NIV. The total duration of NIV use by each subject was $4 \pm 1 \mathrm{~h} / \mathrm{d}$. Subjects reported a wide range of adverse effects, with $20 \%$ having nasal mask intolerance that gradually remitted, $25 \%$ complaining of nasal congestion, and $15 \%$ complaining of gastrointestinal distention due to air insufflations. On average, the NIV setting for IPAP and expiratory positive airway pressure was $11 \pm 3$ and $4 \pm 1 \mathrm{~cm} \mathrm{H}_{2} \mathrm{O}$, respectively. The baseline clinical and physiological data are shown in Table 1. Subjects were divided in 2 subgroups of severe $\left(\mathrm{FEV}_{1}>30 \%\right.$ of predicted) and very severe ( $\mathrm{FEV}_{1}<30 \%$ of predicted) respiratory disease for further analysis (Table 2).

\section{Outcome Measurements}

Clinical and physiological assessments of the 2 subgroups are listed in Table 2. Figure 1 show radiological findings. 


\section{Noninvasive Ventilation in Mustard Airway Diseases}

Table 2. Clinical and Physiologic Data Before and After Study in Severe (FEV $>30 \%$ of predicted) and Very Severe $\left(\mathrm{FEV}_{1}<30 \%\right.$ of predicted) Subjects

\begin{tabular}{|c|c|c|c|c|c|c|}
\hline \multirow{2}{*}{ Clinical and Physiologic Data } & \multicolumn{3}{|c|}{$\mathrm{FEV}_{1}>30 \%$ of predicted $(n=10)$} & \multicolumn{3}{|c|}{$\mathrm{FEV}_{1}<30 \%$ of predicted $(n=10)$} \\
\hline & Baseline & Final & $P$ & Baseline & Final & $P$ \\
\hline BMI, $\mathrm{kg} / \mathrm{m}^{2}$ & $23.6 \pm 5.0$ & $24.0 \pm 4.9$ & .38 & $23.4 \pm 4.0$ & $24.2 \pm 2.8$ & .66 \\
\hline Borg dyspnea scale score & $6.56 \pm 0.88$ & $6.22 \pm 1.48$ & .60 & $6.11 \pm 1.27$ & $5.22 \pm 1.92$ & .29 \\
\hline Visual analog dyspnea scale score & $6.90 \pm 0.82$ & $6.60 \pm 1.04$ & .52 & $7.54 \pm 1.69$ & $6.28 \pm 1.81$ & .07 \\
\hline $\mathrm{FEV}_{1}, \%$ predicted & $37.2 \pm 7.4$ & $32.7 \pm 7.5$ & .08 & $18.9 \pm 4.6$ & $23.2 \pm 8.4$ & .05 \\
\hline Vital capacity, $\%$ predicted & $59.0 \pm 10.6$ & $54.7 \pm 9.6$ & .34 & $40.9 \pm 13.3$ & $45.0 \pm 12.7$ & .29 \\
\hline TLC, $\%$ predicted & $91.1 \pm 9.8$ & $104.4 \pm 14.9$ & .08 & $117.8 \pm 52.2$ & $117.8 \pm 34.5$ & .99 \\
\hline $\mathrm{RV}, \%$ predicted & $171.5 \pm 50.3$ & $227.1 \pm 50.4$ & .06 & $305.7 \pm 130.5$ & $266.0 \pm 60.7$ & .55 \\
\hline RV/TLC, $\%$ predicted & $180.6 \pm 38.4$ & $211.1 \pm 23.9$ & .08 & $240.4 \pm 29.0$ & $242.3 \pm 39.5$ & .95 \\
\hline $\mathrm{P}_{\mathrm{O}_{2}}, \mathrm{~mm} \mathrm{Hg}$ & $62.3 \pm 13.4$ & $59.8 \pm 11.9$ & .60 & $58.1 \pm 16.6$ & $56.2 \pm 7.0$ & .69 \\
\hline $\mathrm{O}_{2}$ saturation, $\%$ & $88.7 \pm 4.6$ & $87.1 \pm 11.1$ & .59 & $85.9 \pm 8.2$ & $87.3 \pm 3.5$ & .52 \\
\hline $\mathrm{P}_{\mathrm{CO}_{2}}, \mathrm{~mm} \mathrm{Hg}$ & $41.4 \pm 5.3$ & $40.3 \pm 3.5$ & .41 & $46.5 \pm 10.2$ & $46.0 \pm 7.5$ & .87 \\
\hline $\mathrm{pH}$ & $7.37 \pm 04$ & $7.35 \pm 01$ & .44 & $7.35 \pm 02$ & $7.37 \pm 02$ & .26 \\
\hline 6MWD, m & $222.2 \pm 123.8$ & $259.1 \pm 161.3$ & .53 & $161.1 \pm 128.4$ & $186.5 \pm 220.0$ & .63 \\
\hline $\mathrm{S}_{\mathrm{pO}_{2}}$ decrease during $6 \mathrm{MWT}, \%$ & $0.91 \pm 1.27$ & $1.80 \pm 1.63$ & .17 & $6.70 \pm 10.29$ & $4.40 \pm 6.50$ & .41 \\
\hline PSQI score & $12.9 \pm 4.4$ & $12.1 \pm 4.6$ & .61 & $12.9 \pm 4.2$ & $11.7 \pm 4.1$ & .06 \\
\hline No. of hospitalizations/y & $2.30 \pm 1.49$ & $0.30 \pm 0.67$ & .00 & $1.89 \pm 1.17$ & $1.89 \pm 1.7$ & $>.99$ \\
\hline No. of ICU admissions/y & 0 & 0 & & $0.56 \pm 1.33$ & $0.33 \pm 1.00$ & .68 \\
\hline No. of intubations/y & 0 & 0 & & $0.0 \pm 0.0$ & $0.30 \pm 0.95$ & .34 \\
\hline No. of exacerbation/y & $4.40 \pm 4.30$ & $1.40 \pm 2.07$ & .01 & $3.00 \pm 3.55$ & $2.13 \pm 2.53$ & .27 \\
\hline \multicolumn{7}{|l|}{ SGRQ score } \\
\hline Symptom & $81.4 \pm 13.5$ & $79.6 \pm 13.2$ & .76 & $75.9 \pm 9.8$ & $63.5 \pm 10.3$ & .02 \\
\hline Activity & $88.2 \pm 11.7$ & $91.1 \pm 12.2$ & .34 & $86.0 \pm 11.9$ & $80.7 \pm 14.7$ & .18 \\
\hline Impact & $78.3 \pm 15.4$ & $72.7 \pm 17.2$ & .45 & $72.8 \pm 14.3$ & $64.9 \pm 14.7$ & .49 \\
\hline Total & $81.5 \pm 10.9$ & $79.5 \pm 10.7$ & .66 & $76.8 \pm 11.5$ & $69.3 \pm 10.3$ & .02 \\
\hline $\begin{array}{l}\text { Data are presented as mean } \pm \text { SD. } \\
\text { BMI }=\text { body mass index } \\
\mathrm{TLC}=\text { total lung capacity } \\
\mathrm{RV}=\text { residual volume } \\
6 \mathrm{MWD}=6 \text {-min walk distance } \\
6 \mathrm{MWT}=6 \text {-min walk test } \\
\text { PSQI }=\text { Pittsburg Sleep Quality Index } \\
\text { SGRQ }=\text { St George Respiratory Questionnaire }\end{array}$ & & & & & & \\
\hline
\end{tabular}

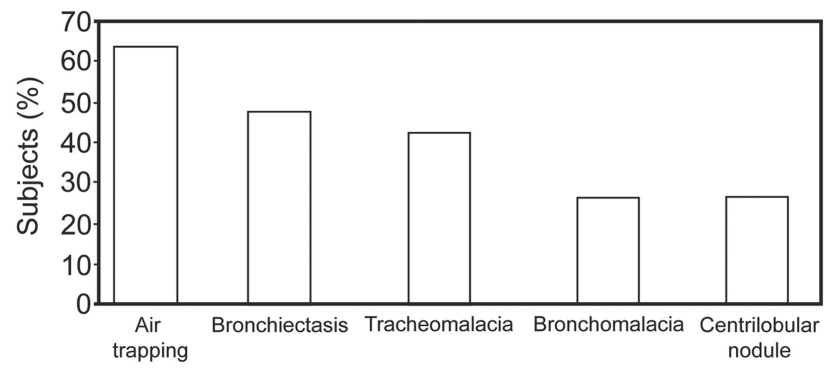

Fig. 1. Inspiratory and expiratory thoracic high-resolution computed tomography imaging findings of subjects.

\section{Discussion}

This is the first study to evaluate the role of domiciliary NIV for subjects with severe obstructive pulmonary disorder due to late complications of sulfur mustard exposure.
Our radiological findings illustrated that our subjects' disease was distinct from classical COPD. We therefore assessed the efficacy of NIV in this particular group of subjects with obstructive pulmonary disease.

We found that NIV improved HRQOL and sleep quality in subjects with very severe mustard airway disease (according to GOLD criteria) and decreased hospitalizations and respiratory exacerbations in subjects with severe mustard airway disease. However, NIV did not significantly improve exercise tolerance, pulmonary function, or dyspnea. The results of subgroup analysis indicated that subjects with very severe disease (according to GOLD criteria) and severe disease had benefited differently from NIV.

Some studies (12 weeks to $2 \mathrm{y}$ in duration) have shown that NIV treatment leads to a significant improvement in HRQOL. ${ }^{17-19}$ Significant improvements in HRQOL total scores in studies reported by Garrod et $\mathrm{al}^{19}$ and Meecham Jones et $\mathrm{al}^{17}$ were associated with improvements in symp- 
tom components of the scores. We found that improvement in the HRQOL total score was directly related to the improvement in respiratory symptoms listed in the $\mathrm{St}$ George Respiratory Questionnaire, such as cough, sputum, wheezing, and dyspnea. When dyspnea was assessed by the visual analog scale, scores improved in very severe subjects (baseline of $7.54 \pm 1.69$, final of $6.28 \pm 1.81$, $P=.07$ ), and this result may help support the notion that improvements in HRQOL are driven by symptom-component score improvement.

Crisafulli et $\mathrm{al}^{20}$ showed that NIV improved subjective sleep quality in subjects with hypercapnic COPD. Similarly, using polysomnography, Krachman et al ${ }^{21}$ demonstrated that the beneficial effects of NIV in subjects with hypercapnic respiratory failure are not solely due to an improvement in gas exchange but may be more complex, with other factors potentially having contributing roles. Our study showed that NIV improved sleep quality $(P=.06)$ in very severe subjects despite no change in gas exchange from a higher baseline $\mathrm{P}_{\mathrm{aCO}_{2}}$ compared with severe subjects. Because better sleep quality is associated with better HRQOL, the improvements observed here have important implications for patient care.

Few studies have assessed hospital and ICU admissions, intubations, and respiratory exacerbations as important outcomes. Two studies reported reductions in hospital admissions in NIV groups compared with long-term $\mathrm{O}_{2}$ therapy groups, but these reductions failed to reach statistical significance. ${ }^{18,22}$ Nevertheless, the reduction in hospital stay was associated with reduced hospital admissions in the NIV groups. In our study, hospitalizations and respiratory exacerbations significantly decreased in the severe subgroup and did not change in the very severe subgroup. Also, the slight increase in intubations in the very severe subgroup was related to 2 subjects who were candidates for lung transplantation, and their disease worsened.

The reduction in hospitalizations due to bi-level NIV use would presumably translate to reduced health-care expenditures, as has already been demonstrated in the setting of acute respiratory failure due to COPD exacerbation. We suspect that this observation is due to the possibility that positive-pressure ventilation with humidification could aid airway clearance in subjects with excessive mucus secretion. ${ }^{23}$ However, our data for hospitalizations have some limitations because prior 3-y admissions and intubations are not based solely on the database, and recall bias could be a problem.

All prior studies evaluating NIV assessed exercise tolerance via the 6-min walk test or shuttle walk test and showed no improvement in the NIV groups, ${ }^{18,19,24-26}$ in agreement with our findings.

Although several NIV studies showed no significant improvement in gas exchange, ${ }^{18,19,21,22}$ Meecham Jones et $\mathrm{al}^{17}$ showed significant improvement in gas exchange, but only by using higher inspiratory pressure in subjects with a higher baseline $\mathrm{P}_{\mathrm{aCO}}$ compared with our study.

There are no studies that show $\mathrm{FEV}_{1}$ improvement with NIV, but one study that included residual volume as an assessment of dynamic hyperinflation reported a significant reduction in residual volume after 3 weeks. ${ }^{27}$ In our subjects with mustard airway disease, we found that NIV decreased functional residual capacity and residual volume non-significantly after 12 months.

A significant reduction in dyspnea was reportedly associated with NIV use in some studies, ${ }^{18,19,22,24}$ but the length of these studies varied greatly, from $5 \mathrm{~d}$ to $2 \mathrm{y}$.

Our study design was limited by its small sample size and potential selection bias. Furthermore, this study was uncontrolled and not blinded to subjects, meaning that the improvements in cases could be attributed to a placebo effect. We could not use high IPAP because of a lack of personnel experience with NIV and its application to mustard airway disease.

The pathophysiology of our subjects' disease in comparison with COPD is critical to understand their differential responses to NIV. For subjects with mustard airway disease, NIV can help reduce respiratory exacerbations and hospitalizations, improve HRQOL, and improve sleep quality. Mustard airway disease is irreversible, with no effective treatment, and consists of asthma (excluded from this study), tracheobronchial stenosis, tracheobronchomalacia, bronchiectasis, bronchiolitis obliterans, and probably COPD. Sulfur mustard can affect the entire set of conducting airways and the small airways in particular. Radiological examination showed that our subjects had complex manifestations of these airway diseases (except tracheobronchial stenosis, which was excluded from this study because it is a contraindication for NIV). Air trapping, a marker of small airway disease and bronchiolitis, was the most common finding in chest high-resolution computed tomography, followed by centrilobular nodules suggestive of bronchiolitis obliterans. To the best of our knowledge, there has been no study on the efficacy of NIV in small airway disease and bronchiolitis. We hypothesize that NIV could be useful for many conducting airway diseases, including small airway disease.

\section{Conclusions}

In summary, our results showed that subjects with very severe mustard airway disease and respiratory failure and those with severe disease experienced different NIV benefits. In subjects with very severe disease and respiratory failure, NIV improved HRQOL and sleep quality, whereas in subjects with severe disease, it reduced exacerbations and hospitalizations. Chest imaging studies showed that these subjects had heterogeneous and complex airway diseases, the main finding of which was bronchiolitis oblit- 


\section{Noninvasive Ventilation in Mustard Airway Diseases}

erans. Therefore, it can be assumed that the different treatment efficacy of NIV could be related to the different severity of airway obstruction or different pathology of mustard airway disease compared with COPD, although this hypothesis requires further exploration and elaboration.

\section{ACKNOWLEDGMENTS}

We thank Dr Mohammadreza Soroush (Director, Janbazan Medical and Engineering Research Center) for funding this research.

\section{REFERENCES}

1. Jones SE, Packham S, Hebden M, Smith AP. Domiciliary nocturnal intermittent positive pressure ventilation in subjects with respiratory failure due to severe COPD: long term follow up and effect on survival. Thorax 1998;53(6):495-498.

2. Kolodziej MA, Jensen L, Rowe B, Sin D. Systematic review of noninvasive positive pressure ventilation in severe stable COPD. Eur Respir J 2007;30(2):293-306.

3. Khateri S, Ghanei M, Keshavarz S, Soroush M, Haines D. Incidence of lung, eye, and skin lesions as late complications in 34,000 Iranians with wartime exposure to mustard agent. J Occup Environ Med 2003;45(11):1136-1143.

4. Beheshti J, Mark EJ, Akbaei HM, Aslani J, Ghanei M. Mustard lung secrets: long term clinicopathological study following mustard gas exposure. Pathol Res Pract 2006;202(10):739-744.

5. Ghanei M, Mokhtari M, Mohammad MM, Aslani J. Bronchiolitis obliterans following exposure to sulfur mustard: chest high resolution computed tomography. Eur J Radiol 2004;52(2):164-169.

6. Ghanei M, Akhlaghpoor S, Mohammad MM, Aslani J. Tracheobronchial stenosis following sulfur mustard inhalation. Inhal Toxicol 2004; 16(13):845-849.

7. Ghanei M, Akbari Moqadam F, Mohammad MM, Aslani J. Tracheobronchomalacia and air trapping after mustard gas exposure. Am J Respir Crit Care Med 2006;173(3):304-309.

8. Ghanei M, Harandi AA. Long-term consequences from exposure to sulfur mustard: a review. Inhal Toxicol 2007;19(5):451-456.

9. American Thoracic Society. Standardization of spirometry, 1994 update. Am J Respir Crit Care Med 1995;152(3):1107-1136.

10. Dubois AB, Botelho SY, Bedell GN, Marshall R, Comroe JH Jr. A rapid plethysmographic method for measuring thoracic gas volume: a comparison with a nitrogen washout method for measuring functional residual capacity in normal subjects. J Clin Invest 1956;35(3): 322-326.

11. American Thoracic Society. Single breath carbon monoxide diffusing capacity (transfer factor). Recommendations for a standard technique. Am Rev Respir Dis 1987;136(5):1299-1307.

12. Cazzola M, MacNee W, Martinez FJ, Rabe KF, Franciosi LG, Barnes PJ, et al. Outcomes for COPD pharmacological trials: from lung function to biomarkers. Eur Respir J 2008;31(2):416-468.
13. Butland RJ, Pang J, Gross ER, Woodcock AA, Geddes DM. Two, six and 12 minute walking tests in respiratory disease. BMJ 1982; 284(6329):1607-1608.

14. Attaran D, Khajedaloui M, Jafarzadeh R, Mazloomi M. Health-related quality of life in subjects with chemical warfare-induced chronic obstructive pulmonary disease. Arch Iran Med 2006;9(4):359-363.

15. Farrahi J, Nakhaee N, Sheibani V, Garrusi B, Amirkafi A. Psychometric properties of the Persian version of the Pittsburgh Sleep Quality Index addendum for PTSD (PSQI-A). Sleep Breath 2009;13(3): 259-262.

16. Ferguson GT, Gilmartin $\mathrm{M} . \mathrm{CO}_{2}$ rebreathing during BiPAP ventilatory assistance. Am J Respir Crit Care Med 1995;151(4):1126-1135.

17. Meecham Jones DJ, Paul EA, Jones PW, Wedzicha JA. Nasal pressure support ventilation plus oxygen compared with oxygen therapy alone in hypercapnic COPD. Am J Respir Crit Care Med 1995; 152(2):538-544.

18. Clini E, Sturani C, Rossi A, Viaggi S, Corrado A, Donner CF, et al. The Italian multicentre study on noninvasive ventilation in chronic obstructive pulmonary disease subjects. Eur Respir J 2002;20(3): 529-538.

19. Garrod R, Mikelsons C, Paul EA, Wedzicha JA. Randomized controlled trial of domiciliary noninvasive positive pressure ventilation and physical training in severe chronic obstructive pulmonary disease. Am J Respir Crit Care Med 2000;162(4):1335-1341.

20. Crisafulli E, Manni G, Kidonias M, Trianni L, Clini EM. Subjective sleep quality during average volume assured pressure support (AVAPS) ventilation in subjects with hypercapnic COPD: a physiological pilot study. Lung 2009;187(5):299-305.

21. Krachman SL, Quaranta AJ, Berger TJ, Criner GJ. Effects of noninvasive positive pressure ventilation on gas exchange and sleep in COPD subjects. Chest 1997;112(3):623-628.

22. Casanova C, Celli BR, Tost L, Soriano E, Abreu J, Velasco V, Santolaria F. Long-term controlled trial of nocturnal nasal positive pressure ventilation in subjects with severe COPD. Chest 2000; 118(6): 1582-1590.

23. Lester MK, Flume PA. Airway-clearance therapy guidelines and implementation. Respir Care 2009;54(6):733-750; discussion 751753.

24. Renston JP, DiMarco AF, Supinski GS. Respiratory muscle rest using nasal BiPAP ventilation in subjects with stable severe COPD. Chest 1994;105(4):1053-1060.

25. Highcock MP, Shneerson JM, Smith IE. Increased ventilation with NiIPPV does not necessarily improve exercise capacity in COPD. Eur Respir J 2003;22(1):100-105.

26. Gay PC, Hubmayr RD, Stroetz RW. Effectiveness of nocturnal nasal ventilation in stable, severe chronic obstructive pulmonary disease during a 3-month controlled trial. Mayo Clin Proc 1996;71(6):533542.

27. Díaz O, Bégin P, Torrealba B, Jover E, Lisboa C. Effects of noninvasive ventilation on lung hyperinflation in stable hypercapnic COPD. Eur Respir J 2002;20(6):1490-1498. 